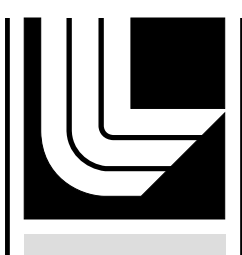

LAW RENCE LIVERMORE N A TIO N A L LABORATORY
Conversion of NIMROD simulation results for graphical analysis using Vislt

C. A. Romero-Talamas

May 5, 2006 
This document was prepared as an account of work sponsored by an agency of the United States Government. Neither the United States Government nor the University of California nor any of their employees, makes any warranty, express or implied, or assumes any legal liability or responsibility for the accuracy, completeness, or usefulness of any information, apparatus, product, or process disclosed, or represents that its use would not infringe privately owned rights. Reference herein to any specific commercial product, process, or service by trade name, trademark, manufacturer, or otherwise, does not necessarily constitute or imply its endorsement, recommendation, or favoring by the United States Government or the University of California. The views and opinions of authors expressed herein do not necessarily state or reflect those of the United States Government or the University of California, and shall not be used for advertising or product endorsement purposes.

This work was performed under the auspices of the U.S. Department of Energy by University of California, Lawrence Livermore National Laboratory under Contract W-7405-Eng-48. 


\title{
Conversion of NIMROD simulation results for graphical analysis using VisIt
}

\author{
Carlos A. Romero-Talamás \\ Lawrence Livermore National Laboratory, \\ 7000 East Avenue, Livermore, California, 94550*
}

(Dated: April 27, 2006)

\begin{abstract}
Software routines developed to prepare NIMROD [C. R. Sovinec et al., J. Comp. Phys. 195, 355 (2004)] results for three-dimensional visualization from simulations of the Sustained Spheromak Physics Experiment (SSPX ) [E. B. Hooper et al., Nucl. Fusion 39, 863 (1999)] are presented here. The visualization is done by first converting the NIMROD output to a format known as legacy VTK and then loading it to VisIt, a graphical analysis tool that includes three-dimensional rendering and various mathematical operations for large data sets. Sample images obtained from the processing of NIMROD data with VisIt are included.
\end{abstract}

\footnotetext{
*Electronic address: romerotalamas1@llnl.gov; The author would like to thank Jeff Moller and Bill Meyer for their help with software and computer issues, to the VisIt support team for their fast response to questions about their software, and to Lynda Lodestro for her comments that helped improve this manuscript. Work performed under the auspices of the U.S. Department of Energy by Lawrence Livermore National Laboratory under Contract W-7405-ENG-48.
} 


\section{INTRODUCTION}

This report describes the procedures and routines used in the conversion of NIMROD [1] simulation results to files readable by VisIt, a visualization and graphical analysis tool package. NIMROD is a three-dimensional (3D) magnetohydrodynamic (MHD) code that is used to simulate plasma dynamics at the Sustained Spheromak Physics Experiment (SSPX) [2]. VisIt is a free (distribution and open source code) package created by the Department of Energy (DOE) Advanced Simulation and Computing Initiative (ASCI) to visualize and analyze large computational data sets. VisIt can read databases written in standard visualization formats and render two or three-dimensional images, as well as perform various mathematical operations on the data [3].

The NIMROD simulation data is stored in binary files called dump files. Each dump file contains information about a particular cycle, that is, a particular time step in the simulation. The nomenclature of each dump file is dump. $X X X X X$, where the five $\mathrm{X}$ represent the digits corresponding to the cycle number (including leading zeroes for numbers smaller than $10^{5}$ ). To visualize the data from a particular dump cycle, a program called NIMPLOT was created by the NIMROD Team[6]. This program takes as input one or more dump files and the user may choose to plot NIMROD variables in different ways, such as vector plots and contour plots, among others. With NIMPLOT, it is also possible to create data output files in the American Standard Code for Information Interchange (ASCII) format. However, all of the plots are two-dimensional, and the user must choose which toroidal location of the simulation domain to plot.

At the time of writing of this report, the files output by NIMPLOT could not be loaded directly into VisIt, and therefore had to be translated into one of the formats recognizable by the visualization program. From these formats, the VTK simple legacy format [4] is relatively simple to work with, and thus a translator was made to rearrange the data in this format. Furthermore, multiple toroidal slices can be included in a single VTK file, conveniently grouping all the necessary data for 3D rendering using VisIt in a single ASCII file. Ideally, dump files would be loaded directly into VisIt to save processing time and storage space. This is indeed possible since VisIt supports database reader plugins. However, the development of a NIMROD dump file plugin for VisIt was not carried out for the present work. 
This document is organized as follows. Section II describes the routines and inputs used to produce volumetric data in separate toroidal slice files by the running NIMPLOT automatically. Section III describes the process of merging toroidal slices to a single volumetric file per time step. The mesh and representation domain inferred by VisIt, along with some examples of 3D rendering and analysis, are shown in Section IV. The complete code and sample inputs used in the conversion process is shown in each corresponding section.

\section{AUTOMATED RUN OF NIMPLOT AND THE CREATION OF DATA SLICES IN ASCII FORMAT}

To convert dump file data to ASCII text files, a group of simple routines was created in Mathematica [5] in order to automate the process of running NIMPLOT and choosing the inputs. These routines take a list of dump cycles and the number of toroidal slices per dump cycle as input, and produce an input file for NIMPLOT. This input file mimics the human input for which NIMPLOT was designed. An excerpt of a NIMPLOT input file (NimplotInput.txt) is shown below.

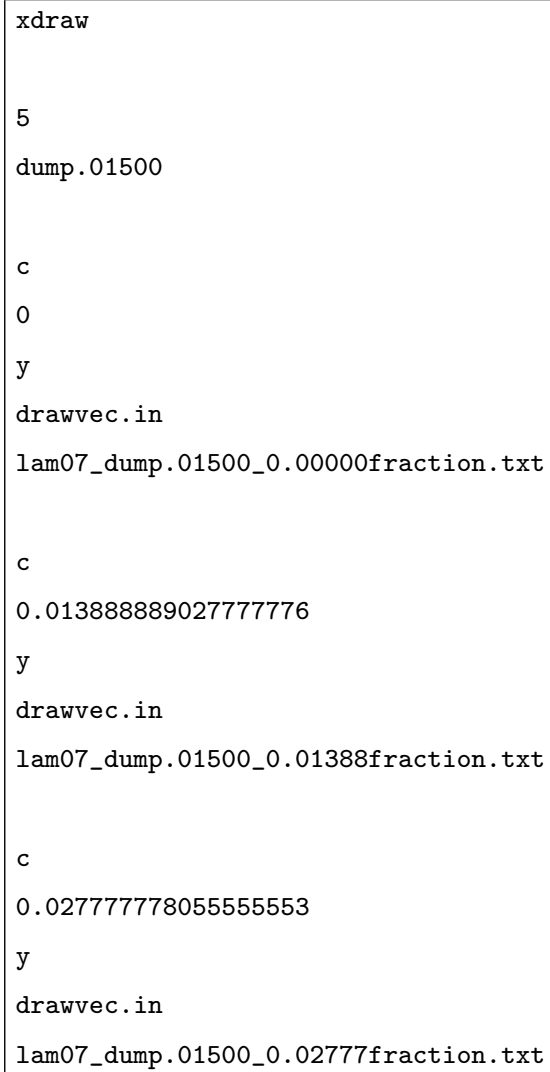


It should be noted that the file above can be created manually and fed into NIMPLOT with the Unix command nimplot < NimplotInput.txt. However, because of the large number of dump cycles and toroidal slices processed, and because Mathematica was installed in grendel.1lnl.gov (the server where the NIMROD dump files are stored), it was more convenient to create the aforementioned routines and run them directly on the server. The code for these routines is shown below. [Note: line numbers are included here only for reference and are not part of the Mathematica file; comments and explanations about the routines are included in enclosed brackets of the form $(*$ some comments ... * $)$, which are not evaluated by Mathematica.]

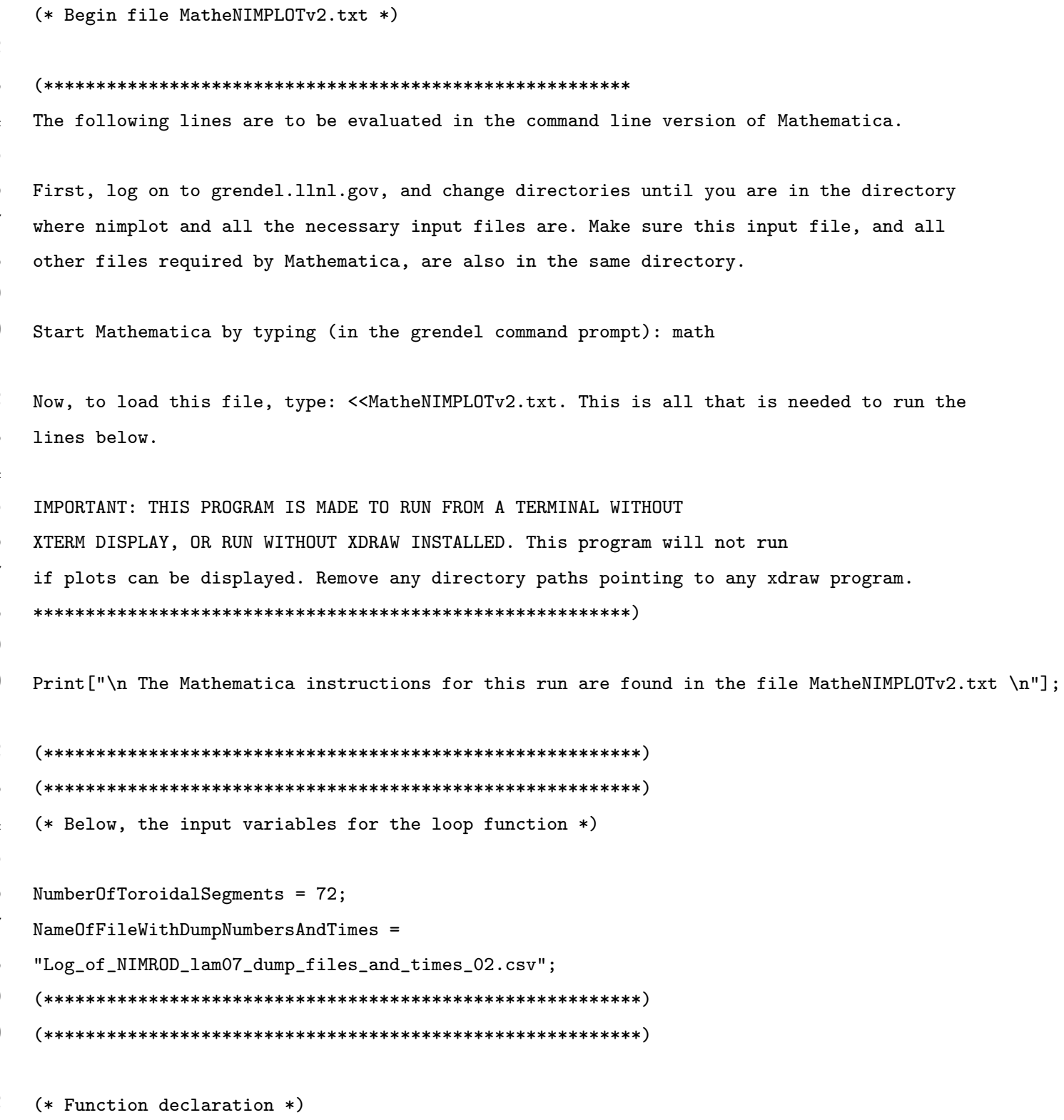




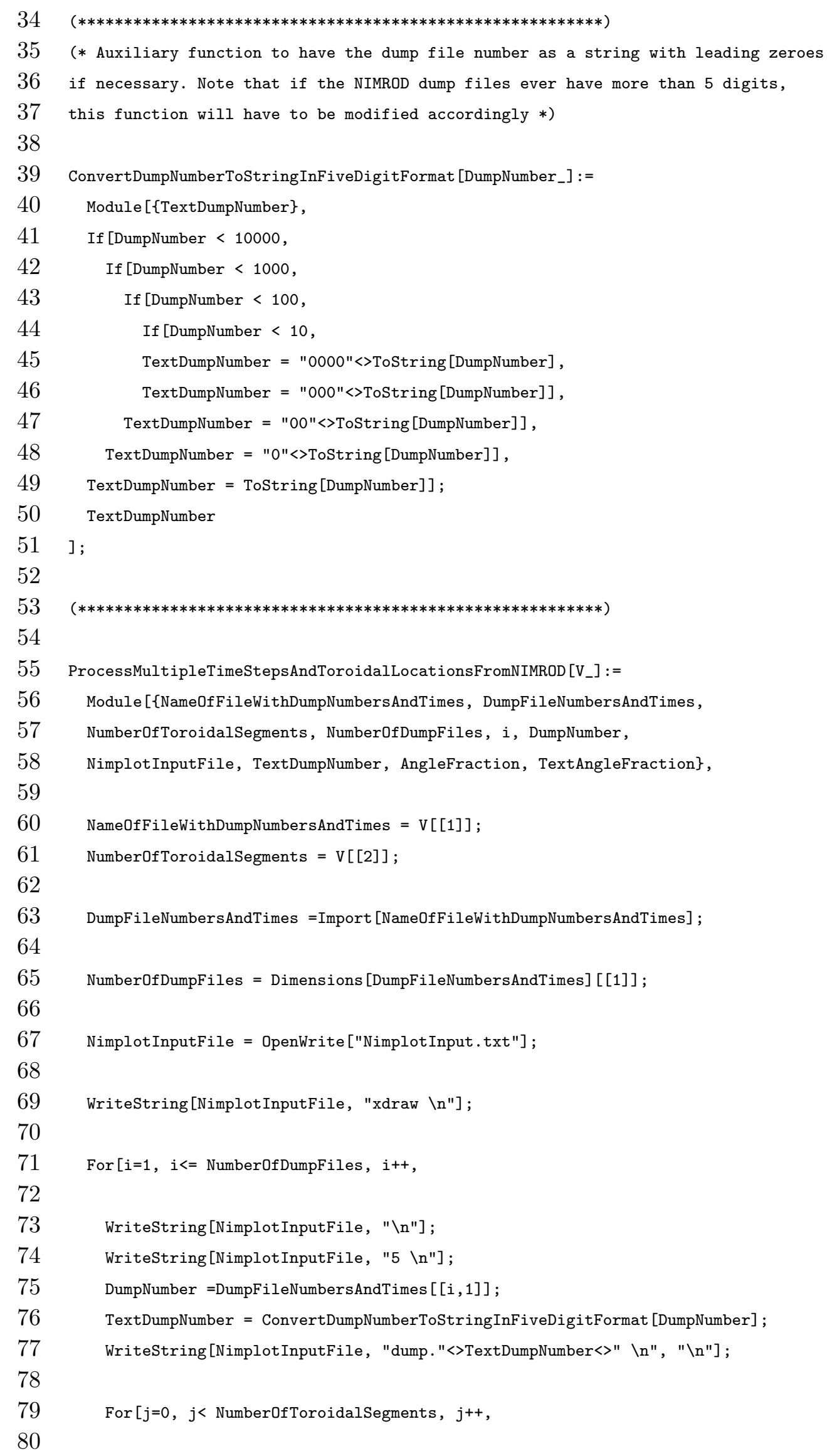

(* Auxiliary function to have the dump file number as a string with leading zeroes 


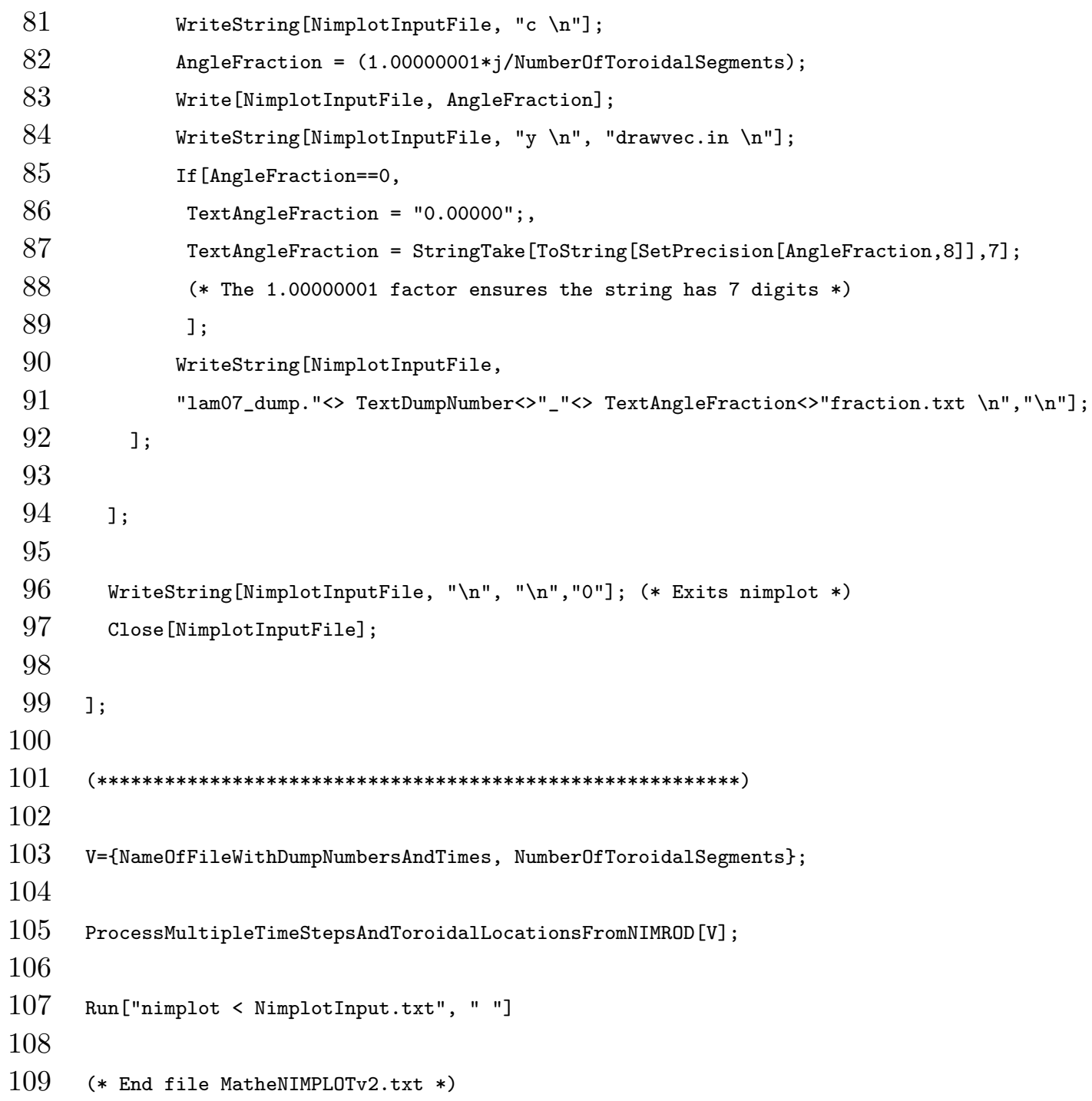

In the program above, between lines 23 and 29, there are two inputs required from the user: the number of toroidal segments in which to divide the simulation domain, and the name of the file containing the dump cycle numbers that the user wants processed including the simulation time they represent. For each dump cycle in the input file, the program would produce 72 text files with the name structure lam07_dump.XXXXX_0.YYYYYfraction.txt, where $\mathrm{X}$ represents the dump cycle number and $\mathrm{Y}$ a fraction between 0 and 1 . The number 72 was chosen in order to maximize the toroidal resolution (in this case every 5 degrees) while having manageable VTK file sizes (less than 100 MB, see Section III) and processing time for a personal computer (tens of minutes per dump cycle).

The file containing the dump cycle numbers and times is written in comma separated value (CSV) format. The number of lines in this file, as well as the particular cycle numbers 
are chosen by the user. Note that the times included in this file are not used for the file processing presented in this report, but are used in other automated processes which will be reported elsewhere. The contents of Log_of_NIMROD_lam07_dump_files_and_times_02.csv are shown below.

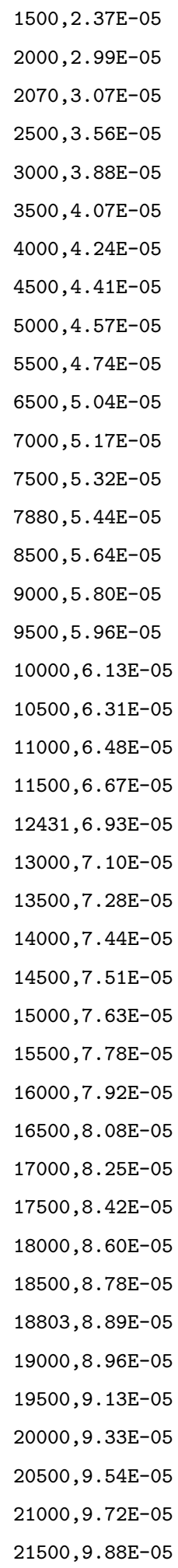




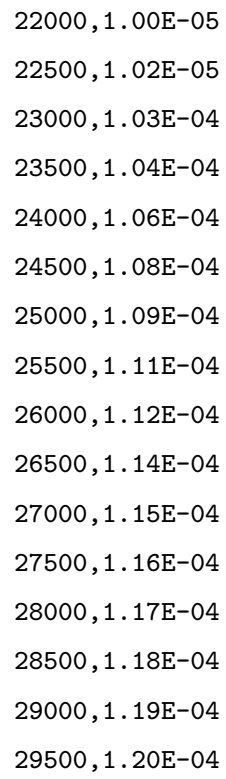

\section{MERGING ALL THE TOROIDAL SLICES INTO A SINGLE VTK FILE}

Once all the required dump cycles have been processed with the routines shown in the previous section, the toroidal slices produced must be lumped into a single VTK file in order to load them easily into VisIt. The routines shown below were created in Mathematica to open the toroidal slice files, extract only one variable of interest, and rearrange the data in the VTK STRUCTURED_GRID format.

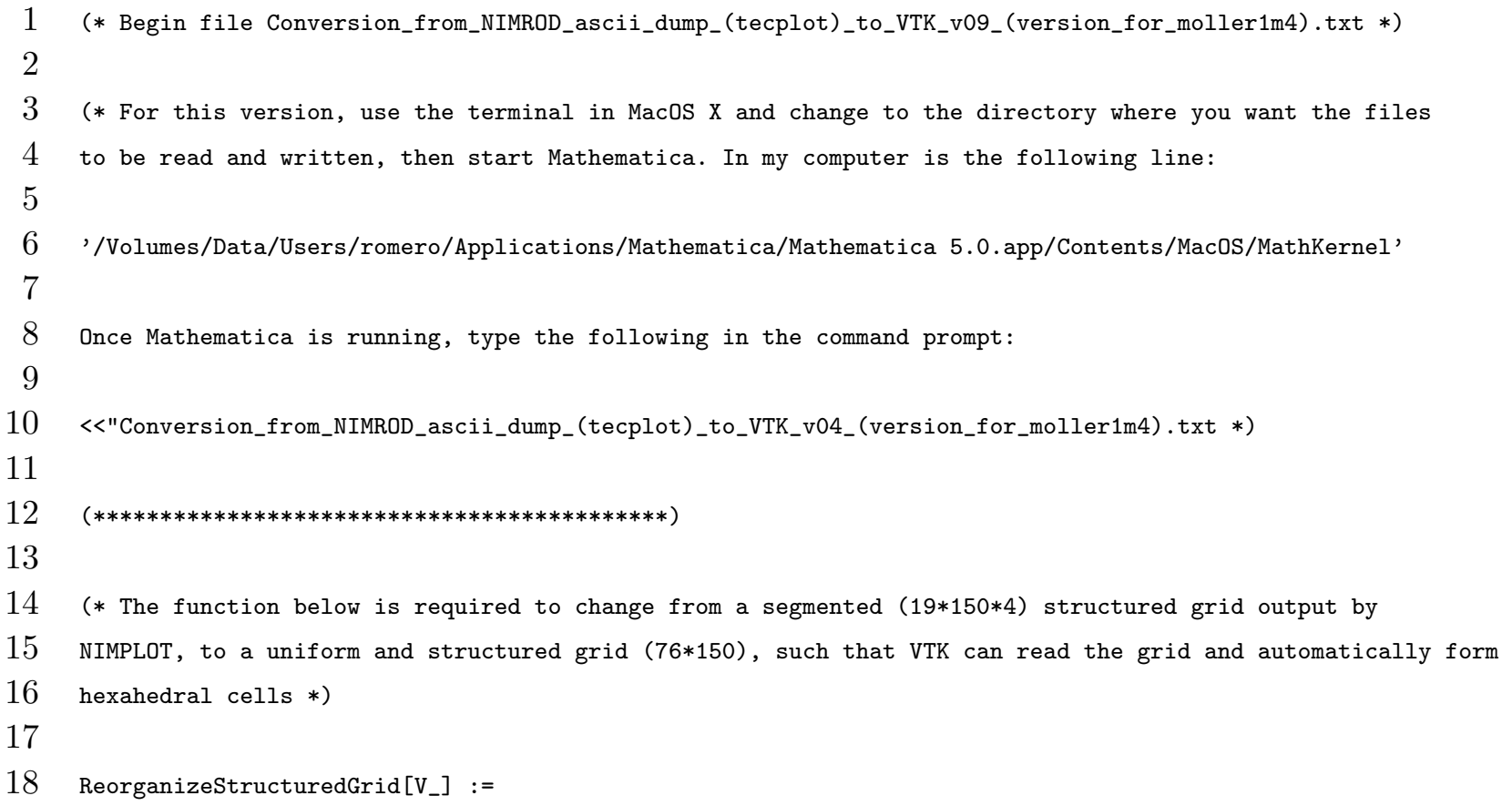


(* The function below assumes that all files contain a three letter extension (e.g. txt) and that the last digits before the extension contain the angle (a fraction between 0 and 1) information and the characters "fraction". 


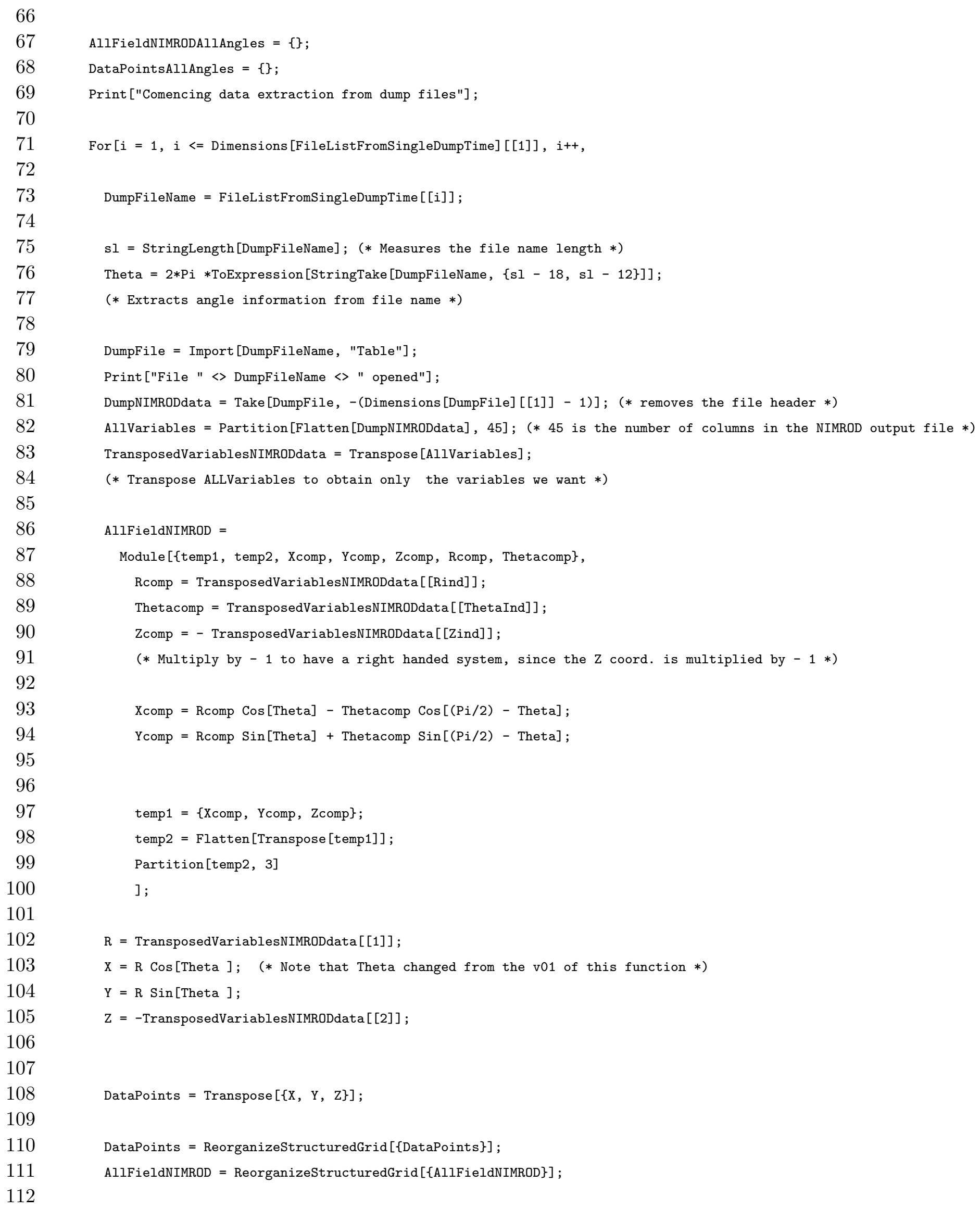




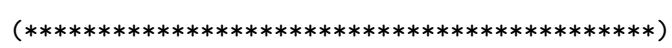




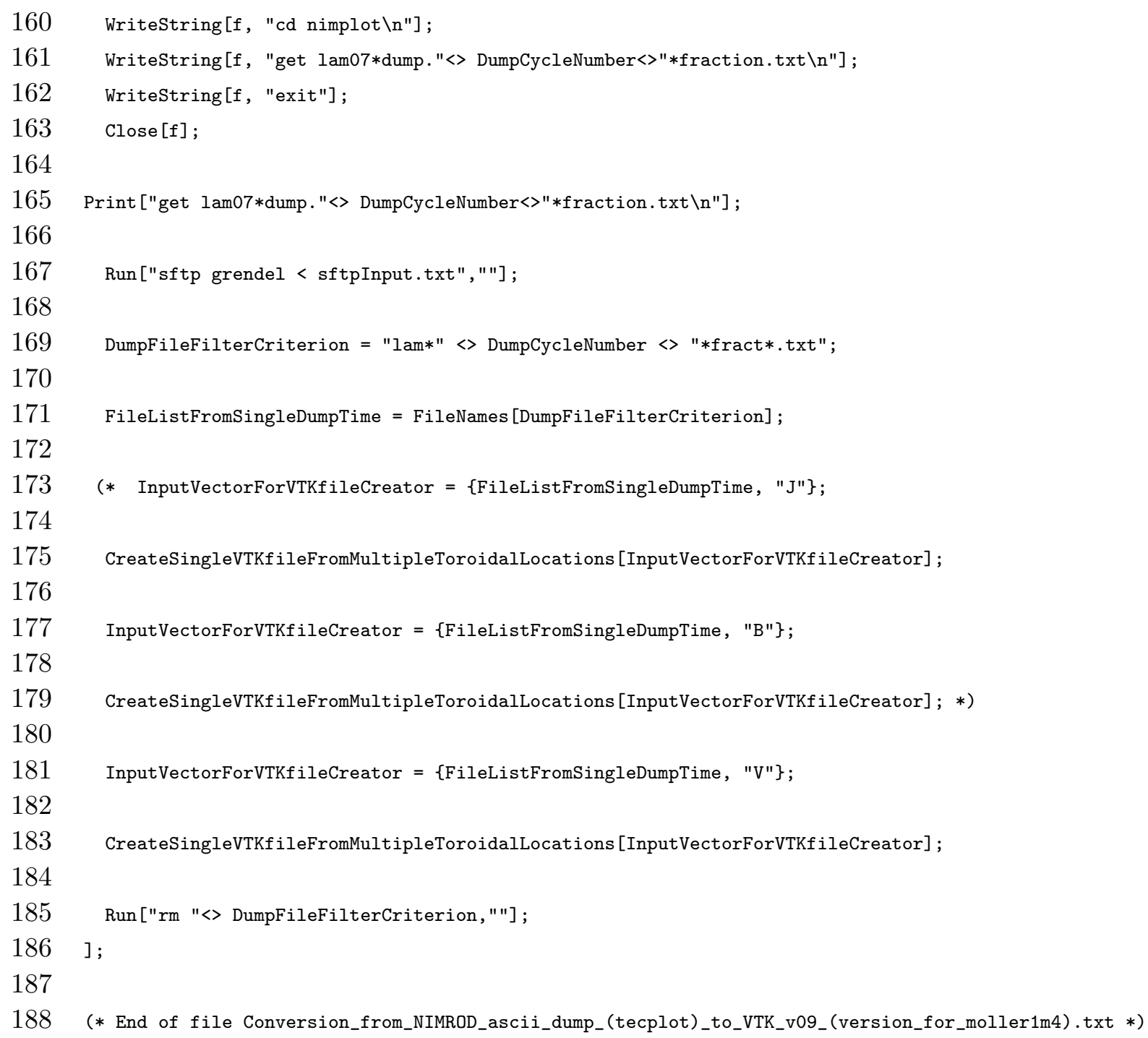

Note that between lines 51 and 65 the choice of variables are the magnetic field B, the current density $\mathbf{J}$, and the fluid velocity $\mathbf{V}$. Any of these variables comprises an array with their three field components. These components are taken from the columns of the NIMPLOT ASCII files. For example, the $B_{r}, B_{\theta}$, and $B_{z}$ components are taken from columns 16, 18, and 17, respectively (shown in lines 52 through 54). If it is desired to obtain scalar variables, the above function would have to be modified considerably to have the option of writing scalar array rather than vector arrays to the VTK file.

The list of dump cycles to process is shown in line 148. The length and content of this list are the choice of the user. The double quotes indicate that each element in the list is a string. Thus, if the same dump cycle list as the one in Log_of_NIMROD_lam07_dump_files_and_times_02.csv is desired, a simple routine that 
extracts the cycle numbers and turns them into strings can be created (using, for example, the function shown in the code of Section II, line 39). Here the list elements were input manually in order to leave the computer process a few elements at a time - typically overnight or over weekends, and commenting out the rest of the list.

Line 167 shows the command for a secure file transfer protocol ( $s f t p$ ) operation with the server where the processed NIMPLOT files are stored. This allows the conversion of files at a terminal rather than the server. For this command to be successful without the intervention of the user at every cycle of the For loop (starting at line 155), passwordless secure shell (ssh) login should be setup beforehand. Alternatively, the sftp operation can be omitted if the conversion and VTK file storage is done in the same server.

The typical size of a VTK file that contains all the toroidal slices from a single dump file is greater than $80 \mathrm{MB}$. The files used here consists of 72 toroidal slices, each with a mesh of $76 \times 150$ data points . An excerpt of one of these VTK files produced with the code described in this section is shown below.

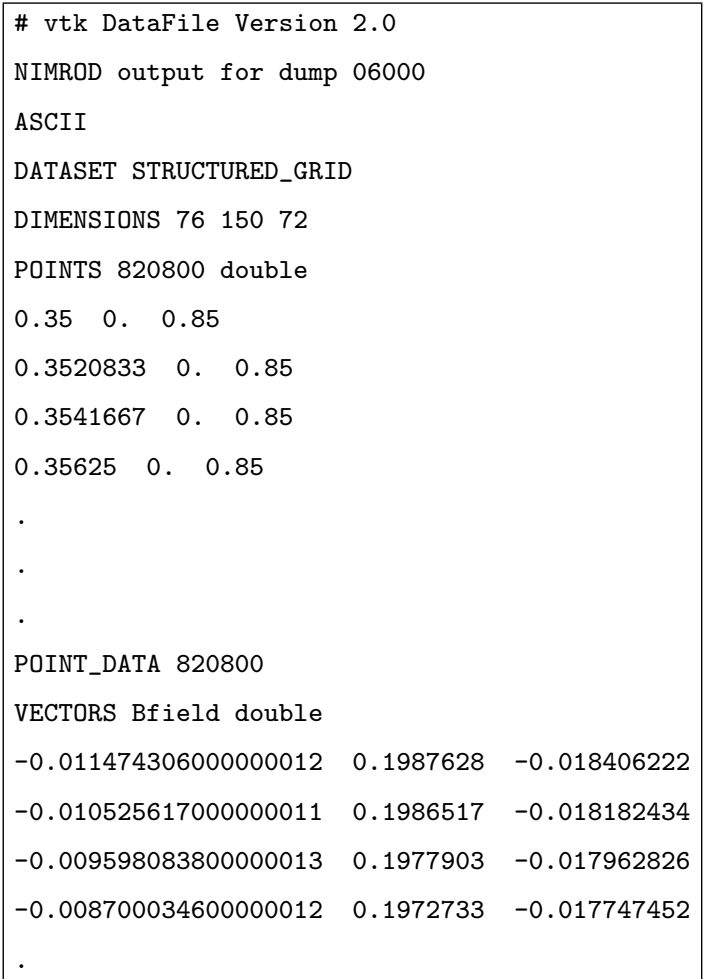

Note how the node $\mathrm{x}, \mathrm{y}, \mathrm{z}$ locations have to be specified first (under the line "POINTS 820800 double") and then vectors corresponding to each node. 


\section{THE MESH RECONSTRUCTED BY VISIT}

One important property of the VTK STRUCTURED_GRID file is that mesh cells are inferred from the layout of the node points in the text file. That is, there is no need to specify the type of cells to be constructed in order to create an interconnected mesh. For the case of the VTK files constructed from 72 slices, the mesh inferred by VisIt is shown in Figure 1. Note the gap in the mesh in frames (b) and (c). This is an unfortunate property of the STRUCTURED_GRID format, since there is no information in the file on how to connect the two ends of the mesh. However, the lack of interpolation available in that gap does not affect the information in the rest of the volume.

Once the mesh is loaded into VisIt, the user can proceed to take advantage of all the plot functions and analysis tools in VisIt. A sample of the processing possible with VisIt is shown in Figure 2.

[1] C. R. Sovinec, A. H. Glasser, T. A. Gianakon, D. C. Barnes, R. A. Nebel, S. E. Kruger, D. D. Schnack, S. J. Plimpton, A. Tarditi, and M. S. Chu. J. Comp. Phys., 195:355, 2004.

[2] E. B. Hooper, L. D. Pearlstein, and R. H. Bulmer. Nucl. Fusion, 39:863, 1999.

[3] Lawrence Livermore National Laboratory, http://www.llnl.gov/visit/manuals.html. VisIt User's Manual, 2003.

[4] Kitware Inc., http://public.kitware.com/VTK/pdf/file-formats.pdf. VTK File Formats.

[5] Stephen Wolfram. The Mathematica Book Online. Wolfram Media, Inc., http://documents.wolfram.com/mathematica/.

[6] http://nimrodteam.org/ 


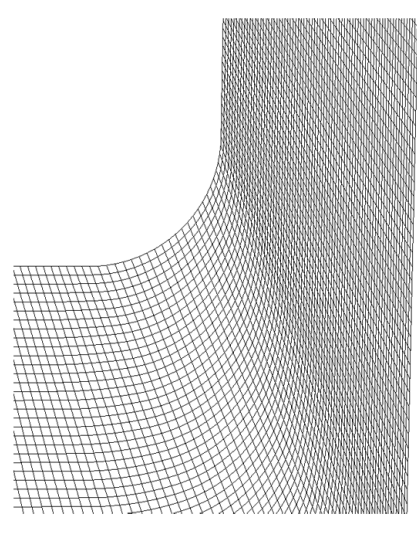

(a)

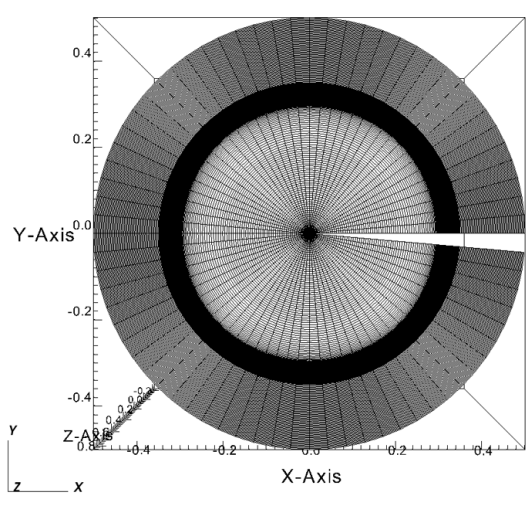

(b)

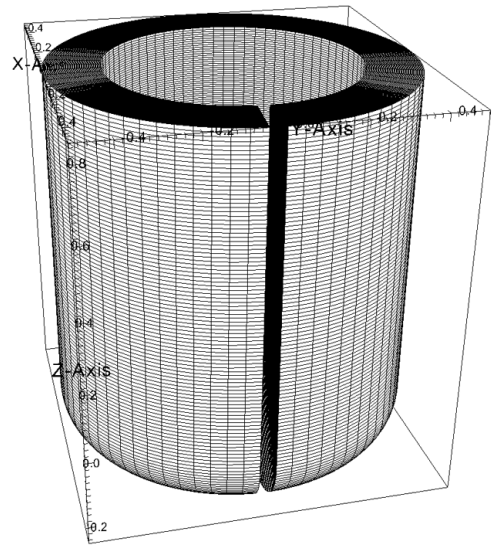

(c)

FIG. 1: Mesh inferred by VisIt from the VTK files. (a) Detail of the mesh. (b) Top view of the 3D mesh. (c) Side view. 

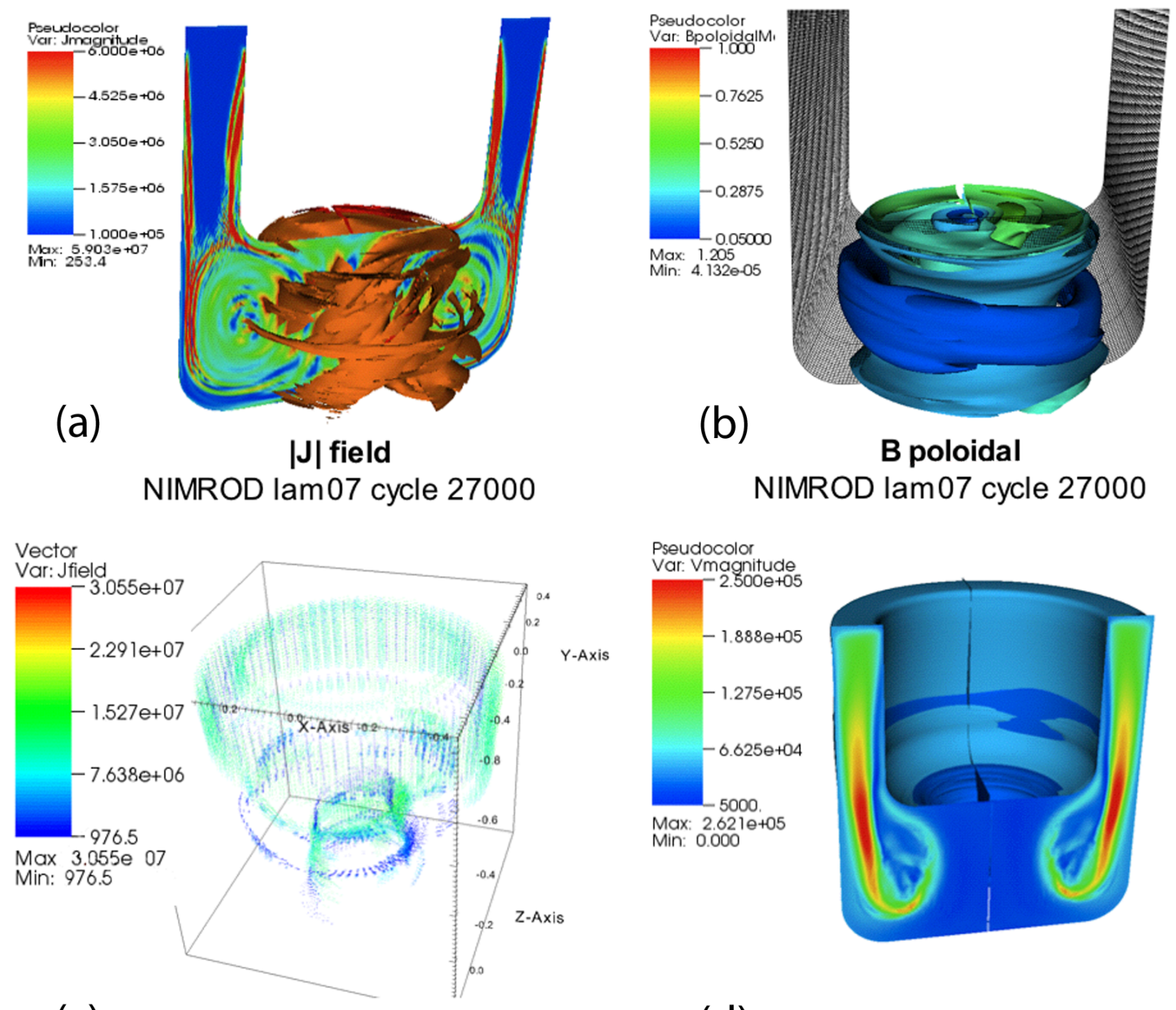

(c)
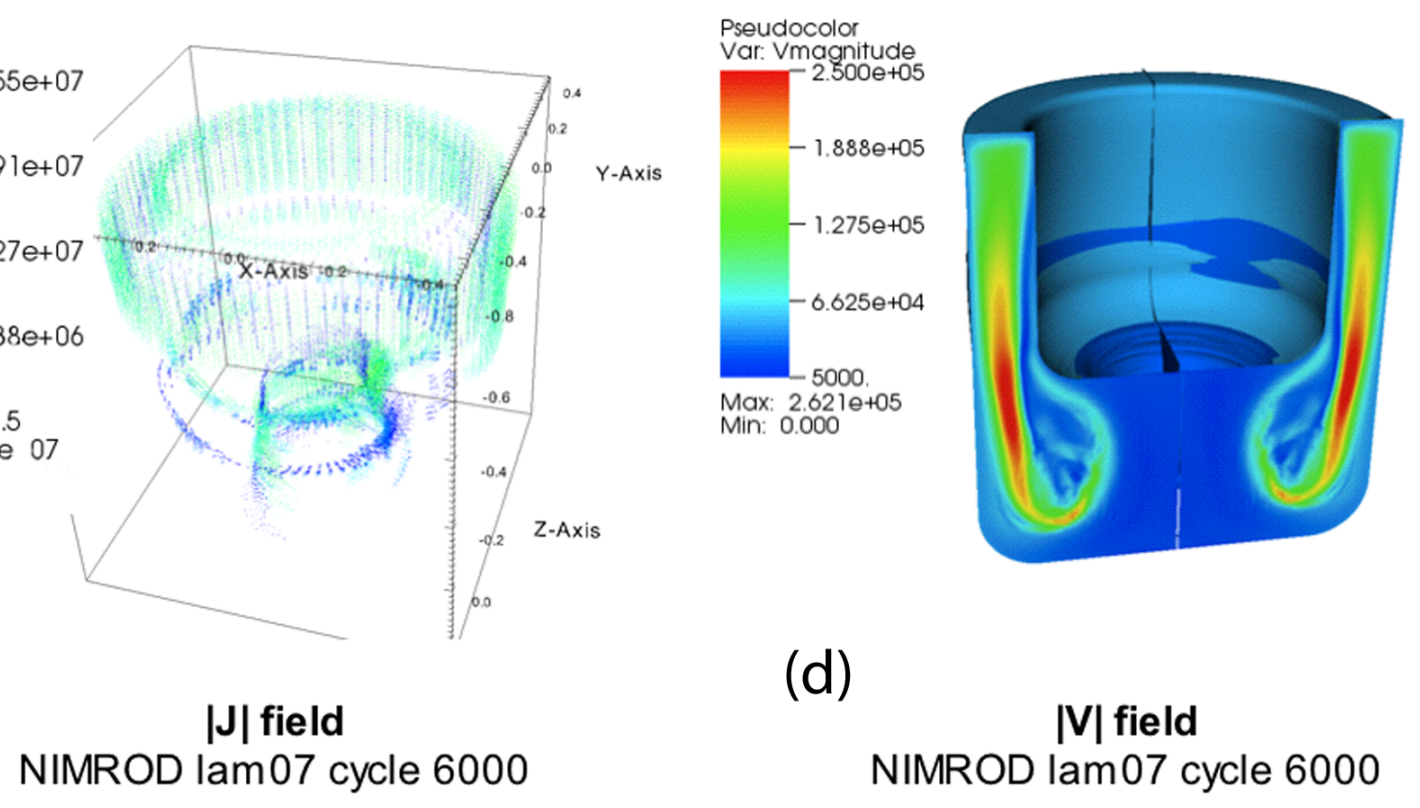

(d)

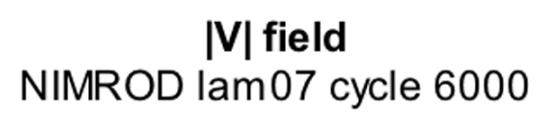

FIG. 2: Sample processing of VTK files with VisIt. (a) Magnitude of the current density J for cycle 27000. The volume plot shows thresholded values of J clipped near the geometrical and magnetic axes. (b) Magnitude of B poloidal (obtained through mathematical operations in VisIt) thersholded and clipped to show only selected values. (c) Thresholded view of J at cycle 6000 showing almost two revolutions in the current path at those values. (d) Magnitude of the velocity field for cycle 6000 . 